\title{
Comparative trachea transcriptome analysis between avian infectious bronchitis virus and avian pathogenic $E$. coli individual infection and co-infection in SPF chickens
}

\author{
S. HASHEMI ${ }^{1}$, A. GHALYANCHILANGEROUDI ${ }^{2 *}$, S. M. HOSSEINI ${ }^{1}$, N. SHEIKHI ${ }^{3}$
}

\begin{abstract}
${ }^{1}$ Department of Microbiology and Microbial Biotechnology, Faculty of Life Sciences and Biotechnology, Shahid Beheshti University, Tehran, Iran; ${ }^{2}$ Department of Microbiology and Immunology, Faculty of Veterinary Medicine, University of Tehran, Tehran, Iran; ${ }^{3}$ Department of Clinical Science, College of Veterinary, Tehran Science and Research Branch, Islamic Azad University, Tehran, Iran
\end{abstract}

\begin{abstract}
Summary. - Avian infectious bronchitis virus (IBV) and avian pathogenic Escherichia coli (APEC) are two important respiratory pathogens in the chicken. The co-infection can lead to chronic complications and considerable economic losses in the poultry industry worldwide. In the present study, we compared differential transcriptional profiles in the trachea tissue of three infected groups (IBV, APEC, and coinfection) with the control group to investigate transcriptome profile changes at the early stage of the infection. After the challenge of SPF chickens with IBV IS-1494 like (GI-23) and APEC, serotype O78: K80, or co-infection, the trachea tissue was used for RNA extraction, and changes in the transcriptome were investigated by Illumina RNA-seq technique. Up-regulated and down-regulated differentially expressed genes (DEGs) in the transcriptome of each group's trachea were identified. Gene ontology category, KEGG pathway, and gene interaction networks (STRING analysis) were analyzed to identify relationships among differentially expressed genes. In general, the numbers of up-regulated genes were higher than of downregulated genes. In the co-infection group, a more severe immune response and macrophage infiltration occurred; an important cluster of pathway signaling in this group's up-regulated genes was an apoptotic cluster, cytokine-mediated signaling cluster, and the PAMPs recognizing cluster. This is the first study to provide a general overview of transcriptome changes in the trachea at the early stage of infection with these pathogens.
\end{abstract}

Keywords: avian infectious bronchitis virus; avian pathogenic E. coli; transcriptome; RNA-Seq

\section{Introduction}

Avian infectious bronchitis (IB) is caused by the IBV, a member of the Coronaviridae family. The virus causes infections mainly in chickens and is a significant pathogen of broilers and laying chickens. IB is an acute, contagious disease characterized primarily by respiratory

"Corresponding author. E-mail: ghalyana@ut.ac.ir, arashghalyanchi@gmail.com; phone: +02161117154.

Abbreviations: APEC = avian pathogenic Escherichia coli; $D E G$ = gifferentially expressed gene(s); FDR = false discovery rate; $\mathrm{IB}=$ avian infectious bronchitis; $\mathrm{IBV}=\mathrm{IB}$ virus; $\mathrm{NLR}=\mathrm{NOD}-$ like receptor; RLR = RIG-I-like receptor; TLR = toll-like receptor symptoms in chickens (Jackwood, 2012). The IS-1494-like IBVs, lineage GI-23, are among the main genotypes in the Middle East (such as Iran) and African countries and were reported from Europe well (Lisowska et al., 2017). IBV is nephropathogenic and affects the respiratory system (Najafi et al., 2016). APEC is responsible for large economic losses in the poultry industry worldwide, causing aerosacculitis, polyserositis, septicemia, and other mainly extraintestinal diseases in chickens (Sandford et al., 2012; Peighambari et al., 2000). The presence of other pathogens, such as IBV and either primary or secondary appearance of APEC respiratory disease in chickens, leads to chronic complicated airsacculitis (Jackwood, 2012; Hynes et al., 2013). Lesions associated with IBV are char- 
acterized by mucosal thickening, loss of cilia, hyperplasia of the surface epithelium, and infiltration of lymphocytes, plasmocytes, and macrophage of lamina propria (Diebold, 2010). The lesion in the trachea associated with APEC is characterized by highly inflamed and congested areas in parabronchi without air in the capillary areas, clogged with exudate, regions containing fewer erythrocytes, and a higher number of lymphocytes, macrophages, and heterophils (Horn et al., 2012).

Infection initiates with a dynamic cascade of events that culminates in altered gene expression patterns in both host and interacting organisms. On the host side, the different expression patterns of immune response genes were shown, and many genes were especially expressed during the intracellular-development stage, which seems to be among the most highly regulated compared to other groups of genes (Westermann et al., 2012).

The first line of defense against pathogens is physical barriers and mucous membranes, complements, antimicrobial peptides, and leukocyte subsets such as heterophils, macrophages, and NK cells, basophils, and eosinophils, that are a subset of the innate immune response. Dendritic cells (DCs), macrophages, lymphocytes, endothelial cells, and fibroblasts present PRRs (pattern recognition receptor) such as TLRs, RLRs, and NLRs cytoplasmic surfaces, through which they can recognize infectious agents. TLR1 LA, TLR1 LB, TLR2A, TLR2B, TLR3, TLR4, TLR5, TLR7, TLR15, and TLR21 are known in chickens (Paul et al., 2013). After activation of the innate immune response, phagocytic heterophils and macrophages are recruited to the infection (Cook et al., 2012).

The inflammatory cytokines and chemokines are among the important regulators of both innate and adaptive immune systems that initiate cascades of intracellular signaling for specific cell functions (Kruth, 1998). The increase of their concentration has been recorded during IBV infection, which leads to the production of acute-phase proteins (APP) in chickens (Asif et al., 2007). Also, increased expression of IL2, IL4, IL7, IL9, IL15, and IL21 was previously shown in the tracheal tissue during infection of chickens with pathogens (Guo et al., 2008).

Virus replication is limited through host antiviral responses such as apoptosis or agents that can induce cell death following target cells' infection. The adaptive immune response through $B$ cells, $T$ cells, macrophages, and leucocytic memory cells' production plays an important role in IBV infection (Chhabra et al., 2015).

One of the new approaches to studying changes in gene expression during host-pathogen interactions is the RNA-seq technique that elucidates the molecular mechanisms of pathogenesis and the corresponding immune response (Westermann et al., 2012). There are few studies regarding the immunological mechanisms involved dur- ing the pathogenesis of IBV and APEC. This study aimed to compare differential immune response after infection SPF chickens with IBV and APEC individually and after co-infection.

\section{Material and Methods}

Virus and bacterial strains. IS-1494 like (GI-23) avian infectious bronchitis virus obtained from the University of Tehran's virus collection was used in this study. The virus was isolated in 2016 from broiler flocks that showed typical clinical signs such as depression, ruffled feathers, nasal discharge, and coughing. Avian pathogenic E. coli serotype 078: K80 from the University of Tehran's culture collection was used for this study. The strain's stock cultures were held in Luria Bertani broth (LB) and $15 \%$ glycerol at $-80^{\circ} \mathrm{C}$.

Chicken challenge and tissue collection. Eighty SPF chickens were divided into four equal groups consisting of three groups of twenty chickens as experimental groups and twenty chickens as a control group. All groups were kept in separate isolators. For the challenge, 12-day-old chickens were inoculated with $100 \mu \mathrm{IBV}\left(10^{4} \mathrm{EID}_{50}\right)$ oculonasally in the first group and with $100 \mu \mathrm{l}$ APEC suspension $\left(1 \times 10^{8} \mathrm{cfu} / \mathrm{ml}\right.$ in PBS $)$ intratracheally in the second group. The third treatment group received $100 \mu \mathrm{l}$ IBV oculo-nasal, followed by $100 \mu \mathrm{l}$ APEC intratracheally 72 hours later. Control groups were inoculated with $100 \mu \mathrm{l}$ PBS.

Table 1. Primers used in qRT-PCR

\begin{tabular}{ll}
\hline Primer name & Sequence \\
\hline TLR4 & GTCTCTCCTTCCTTACCTGCTGTTC \\
& AGGAGGAGAAAACAGGGTAGGTG \\
& GGAAACGCTACTAACCTG \\
TLR2-2 & GTTTGCTTCCAGACTCAG \\
& AGGCACTTGAGATGGAGCAC \\
MyD88 & CCTGTTATGGGCCAGGTTTA \\
& AGAAGGTGTCGGAGGATGGTG \\
TRAF6 & GGGCTCCAAATGCTGACTGC \\
& GAGTGTCCAAGGCGTCAAGTCTG \\
IL1R2 & GTGTCGTGCCAGTTCATTCCTC \\
& AACTGCCCAGATCACACCAT \\
CSF3R & TCAAGGCAGAAAAATCACAT \\
& CCTCTTCGGGACGCTGTA \\
IL8L1 & GGGCTTTCTCATGGGTAGTG \\
& CCTCACTGCAAGAATGTGGAAG \\
IL18 & TATTGGTGTCTGCCTTGTCAG \\
& AGGAGGTGAAATCTGGCAGTG \\
TGFBR1 & TCTTCTACCTGGACGCTGAATG \\
& TGAGTATTGGGCCAAAGAGCTG \\
GAPDH & ACACGAAGAAGATGCTGTGG \\
& TGCTGCCCAGAACATCATCC \\
& ACGGCAGGTCAGGTCAACAA \\
\hline
\end{tabular}


(a)

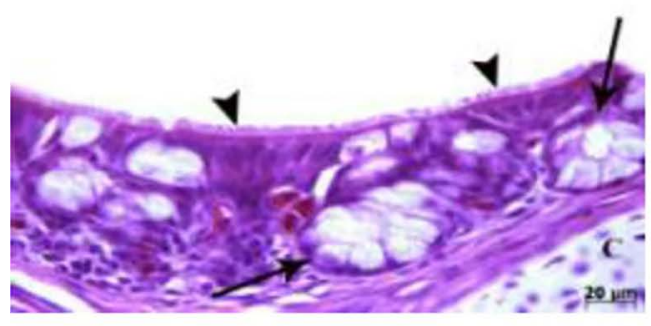

(c)

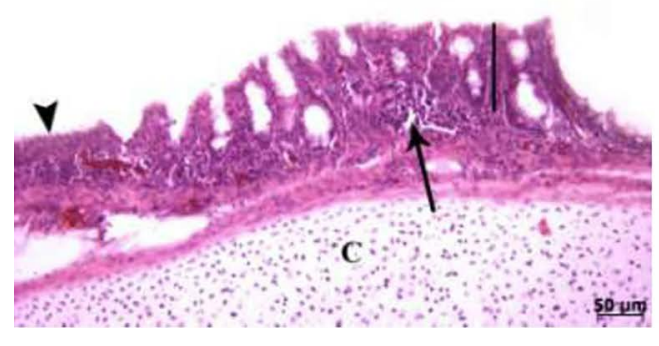

(b)

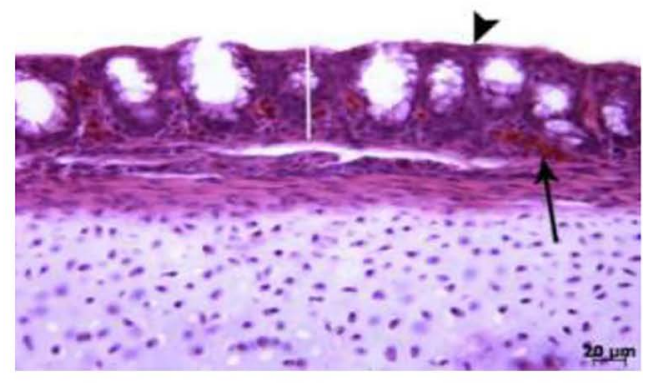

(d)

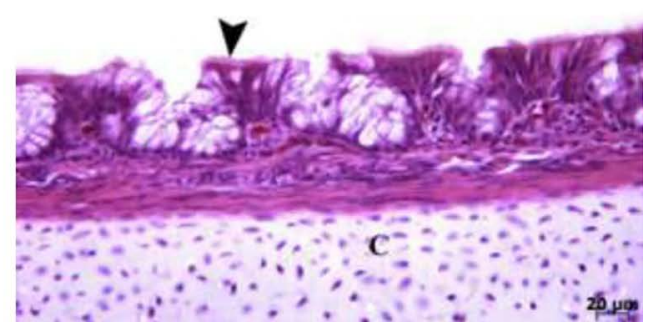

Fig. 1

Histological section of the trachea of experimentally infected chickens

(a) Chicken challenged with IBV (IS-1494 like; GI-23) virus, the respiratory trachea's epithelium was pseudo-ciliated with mucous membranes (simple bubbles) inside the epithelium covered. (b) Chicken challenged with APEC. There is the loss of cilia, a moderate increase of epithelial thickness because of hyperplasia of epithelial cells, and vascular hyperemia. (c) Chicken challenged with co-infection. Increased epithelial thickness because of hyperplasia of epithelial cells, loss of cilia in epithelial cells, infiltration of inflammatory cells of lymphocytes in lamina propia with severe vascular hyperemia. (d) Uninfected chicken. A monolayer of epithelial cells covers the mucosa.

Chickens were euthanized at three days post-infection, and the tracheal organs were harvested. A piece of the trachea at 3 days post-infection from control and each challenged group were collected and added directly to the RNAlater solution (Thermo Fisher Scientific) and transferred to $-80^{\circ} \mathrm{C}$. A part of the trachea sample from each challenge group and the control group was kept for the qPCR validation of the RNA-seq results for IBV detection and culture for APEC)s colony count.

Histopathological examination. Tracheal tissue was fixed in $10 \%$ formalin and examined histopathologically. Sections were stained with hematoxylin and eosin ( $\mathrm{H} \& \mathrm{E})$ and, based on the histopathological examination, were evaluated for the presence of typical microscopic lesions and the presence of the infiltration of inflammatory cells.

RNA extraction. Total RNA was extracted from both control samples and challenged groups using TRIzol reagent (Thermo Fisher Scientific) according to the manufacturer's instructions. On-column DNA digestion was performed using RNase-free DNase (SinaClone) to remove contaminating genomic DNA. The amount of RNA was quantified using an Agilent Bioanalyzer 2100. Total RNAs were subjected to RNA-seq and transcriptome analysis.

Illumina library construction, RNA sequencing, and analysis. Acquired data of Illumina HiSeq 2500 platform were processed to obtain raw reads, which were then extracted for quality control and read mapping. DEGs were identified using CLC Biomedical software (Trial version) and were aligned using the reference genome (Gallus Gallus reference) extracted from NCBI. DEGs were analyzed by comparing the gene expression level between the infected and non-infected groups. Database for annotation, visualization, and integrated discovery analysis was used to perform functional annotation based on the GO (Gene Ontology) database (FDR <0.05), and pathway enrichment analysis based on the KEGG (Kyoto Encyclopedia of Genes and Genomes) database (FDR <0.05) was used to explore the various biological processes. Then, gene interaction networks were analyzed using the STRING database of known and predicted protein interactions, which included direct (physical) and indirect (functional) associations, and the PPI networks were visualized.

Validation of RNA-seq data by qRT-PCR. For validation of the RNA-seq data, total RNA samples in the group infected with IBV and APEC were analyzed by quantitative real-time PCR (qRTPCR). Briefly, $1 \mu \mathrm{g}$ of RNA samples was reverse-transcribed into cDNA using the cDNA synthesis kit (Thermo Fisher Scientific). Ten up-regulated genes were selected, including TLR4, TLR7, TLR2-2, MyD88, TRAF6, IL1R2, CSF3R, IL8L1, TGFBR1, and IL18. All qRT-PCR primers were designed using Primer-BLAST 


\begin{tabular}{l}
\hline GO Biological Process \\
cellular protein modification process (G0:0006464) \\
cotranslational protein targeting to membrane (G0:0006613) \\
phosphorylation (G0:0016310) \\
protein targeting to ER (G0:0045047) \\
cellular protein metabolic process (G0:004426) \\
nuclear-transcribed mRNA (atabolic process (G0:0000956) \\
regulation of translation (G0:0006417) \\
protein phosphorylation (G0:0006468) \\
nuclear-transcribed mRNA (catabolic process, nonsense-mediated decay (G0:0000184) \\
viral process (G0:0016032)
\end{tabular}

GO Molecular Function

RNA binding (G0:0003723)

cadherin binding (G0:0045296)

phosphatidylinositol monophosphate phosphatase activity (60:0052744)

phosphatidylinositiol-3-phosphatase activity (60:0004438)

kinase activity [G0:0016301)

protein serine/threonine kinase activity (GO:0004674)

adenyl ribonudeotide binding (60:0032559)

phosphatidyl| nositol-3,5-bisphosphate phosphatase activity (G0:0106018)

GTPase regulator activity (G0:0030695)

viral process (G0:0016032)

GTPase activator activity (G0:0005096)

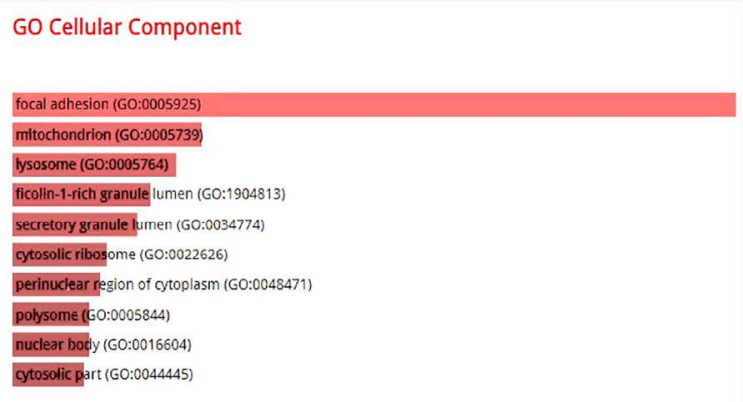

Fig. 2

GO analysis of differentially expressed genes

DEGs in the group infected with APEC were categorized into the three main GO categories, including biological process, cellular component, and molecular function, that were significantly enriched (FDR $<0.05)$.

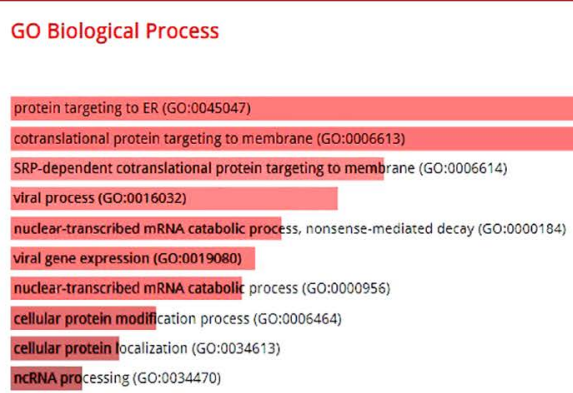

GO Molecular Function

RNA binding (G0:0003723)

cadherin binding (G0:0045296)

phosphatidylinositol monophosphate phosphatase activity (GO:005274

phosphatldylinositol-3-phosphatase activity (G0:0004438)

adenyl ribonucleotide binding (GO:0032559)

purine ribonucleoside triphosphate binding (G0:0035639)

phosphatidy linositol-3,5-bisphosphate phosphatase activity (G0:0106018)

ATP binding (GO:0005524)

double-stronded RNA binding (G0:0003725)

kinase activity $\{60: 0016301\}$

GO Cellular Component
focal achesion (G0:0005925)
cytosolic part (G0:0044445)
|ysosome (G0:0005764)
cytosolic ribosome (G0:0022626)
perinuclear region of cytoplasm (G0:0048471)
ficolin-1-rich granule lumen (G0:1904813)
secretory granule lumen (GO:0034774)
mitochondrion (GO:0005739)
small ribosomal subunit (G0:0015935)
ribosome jG0:0005840)

Fig. 3

GO analysis of differential expression genes

DEGs in the group infected with IBV were categorized into the three main GO categories, including biological process, cellular component, and molecular function, that were significantly enriched (FDR <0.05). 


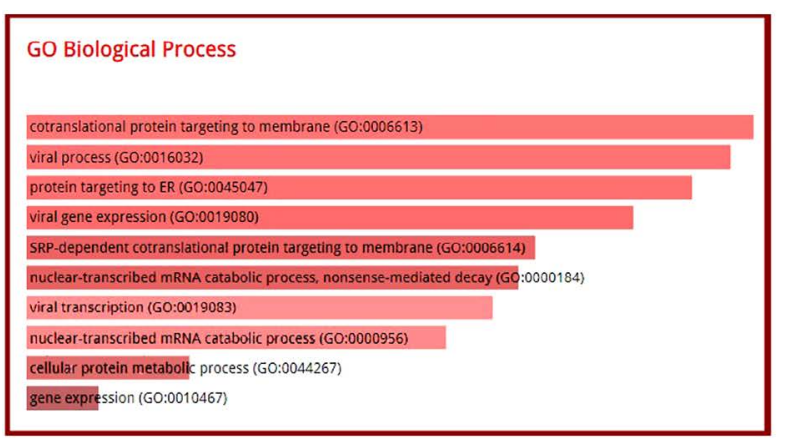

Go Molecular Function

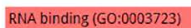

cacherin binding (G0:0045296)

purine ribonucleoside triphosphate binding (G0:0035639)

kinase activity (G:0016301)

ATP binding (G0:0005524)

kinase binding [60:0019900)

adenyl ribonucleatide hinding (G0:0032559)

protein serinerthreonine kinase activity (G0:0004674)

protein kinase activity (G0:0004672)

double-stranded RNA binding (GO:0003725)

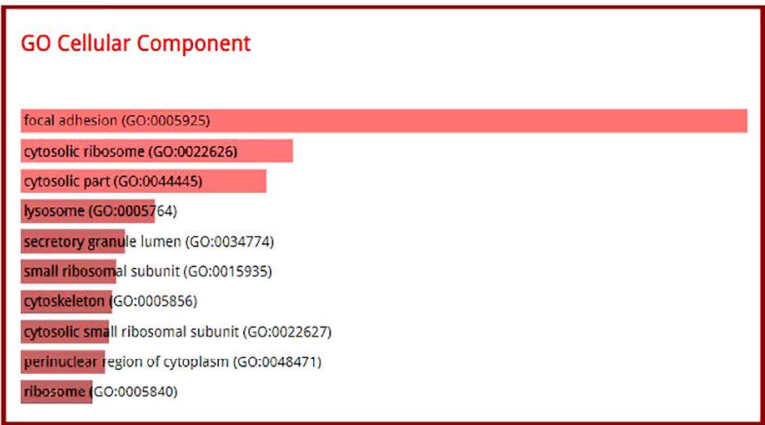

Fig. 4

GO analysis of differential expression genes

DEGs in the co-infected group were categorized into the three main GO categories, including biological process, cellular component, and molecular function, that were significantly enriched (FDR <0.05).

(https://www.ncbi.nlm.nih.gov/tools/primer-blast/) and are listed in Table 1. QRT-PCR was performed using the qPCR Green Master Kit (Jena Bioscience) according to the manufacturer's instructions. The PCR conditions consisted of an initial denaturation at $95^{\circ} \mathrm{C}$ for $30 \mathrm{~s}$, followed by 40 PCR cycles of $95^{\circ} \mathrm{C}$ for $20 \mathrm{~s}, 60^{\circ} \mathrm{C}$ for $30 \mathrm{~s}$, and $72^{\circ} \mathrm{C}$ for 30 s using Rotor-Gene $\mathrm{Q}$ Real-Time PCR (Qiagen). Relative expression of target genes was analyzed by the $2^{-\Delta \Delta C t}$ method, and GAPDH was used as an endogenous housekeeping gene for normalizing expression levels. All samples were analyzed in triplicate.

\section{Results}

\section{Clinical and histopathological changes}

In the group of chickens infected with APEC and IBV individually, no clinical signs were observed, and in the co-infected group, depression, sneezing, and exudate in the eyes were observed. No mortality rate was observed in any treatment group.

In the control group, normal epithelial cells with mucosa were observed, and in IBV and APEC individual groups, loss of cilia and vascular hyperemia was observed. A severe pathological effect was observed in the co-infected group, with the infiltration of inflammatory cells in lamina propia (Fig. 1).

\section{Functional analysis of DEGs}

Illumina HiSeq 2500 platform, paired-end, and strandspecific sequencing produced an average read length of $\sim 150 \mathrm{bp}$. The average number of reads obtained was $\sim 113$ million reads per sample. After the statistical analysis with CLC Biomedical software, DEGs were analyzed by comparing gene expression levels between the infected and non-infected groups. In the group infected with IBV, 5002 DEGs were identified in trachea transcriptome, of which 3453 were up-regulated and 1549 down-regulated. In the group infected with APEC, 4964 DEGs were identified in trachea transcriptome, of which 3550 were upregulated and 1414 down-regulated. In the co-infected group, 5011 DEGs were identified in trachea transcriptome, of which 3599 were up-regulated and 1412 down-regulated. The GO database classifies genes according to the three categories: biological process, cellular component, and molecular functions, and then predicts the function of the selected genes. Significantly enriched GO categories in the DEGs in each group are shown in Figs. 2, 3, and 4 (FDR <0.05). 
(a)

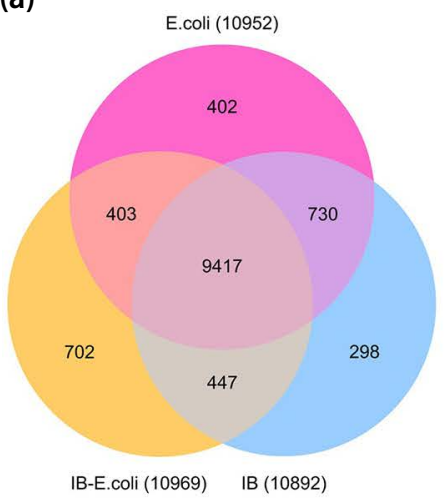

(b)

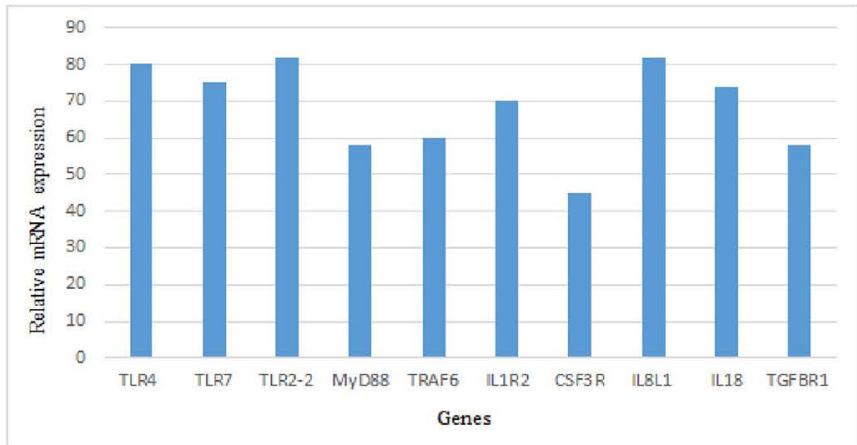

(c)

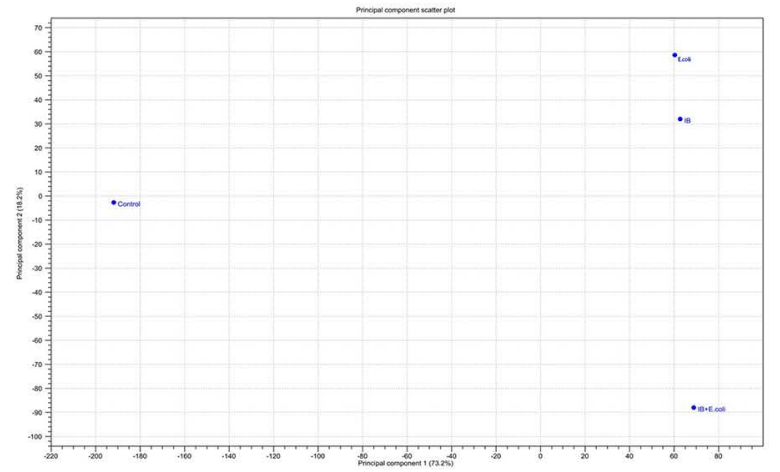

(d)

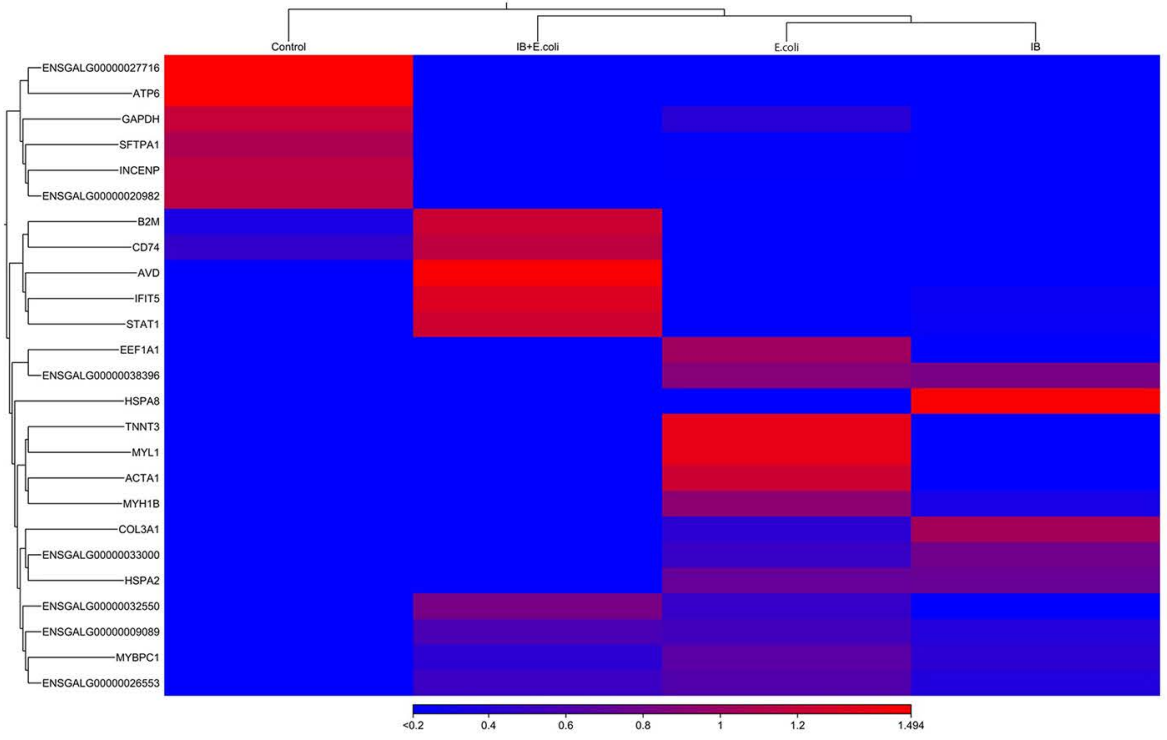

Fig. 5

(a) Venn diagram illustrating the overlap of DEGs among the three groups. (b) qRT-PCR confirmation of 10 randomly selected mRNAs from the up-regulated genes in the co-infected group. (c) PCA diagram of DEGs among the three groups. (d) The heat map analysis of genes showed significant changes and relative gene expression. 
Table 2. KEGG pathway analysis for down-regulated differentially expressed genes

\begin{tabular}{lllll}
\hline A & ID & KEGG pathway-description & Observed gene count & FDR \\
\hline & gga03010 & Ribosome & 50 & $1.26 \mathrm{E}-18$ \\
& gga03040 & Spliceosome & 22 & 0.0032 \\
\hline B & ID & KEGG pathway-description & Observed gene count & FDR \\
\hline & gga03010 & Ribosome & 55 & $1.84 \mathrm{E}-21$ \\
& gga03040 & Spliceosome & 23 & 0.002 \\
& gga00190 & Oxidative phosphorylation & 23 & 0.0029 \\
\hline C & ID & KEGG pathway-description & Observed gene count & FDR \\
\hline & gga03010 & Ribosome & 67 & $1.81 \mathrm{E}-31$ \\
& gga03040 & Spliceosome & 24 & 0.0003 \\
& gga00190 & Oxidative phosphorylation & 24 & 0.00047 \\
& gga00480 & Glutathione metabolism & 11 & 0.0142 \\
& gga01100 & Metabolic pathways & 109 & 0.02 \\
gga04260 & Cardiac muscle contraction & 12 & 0.0306 \\
\hline
\end{tabular}

Pathway analysis enrichment using DEGs between A: control and the group of infected with APEC, B: control, and infected with IBV and C: control and co-infected group. FDR $<0.05$ was used as a threshold to select significant KEGG pathways.

Venn diagrams were used for illustrating the relationship between the three treatment groups using gene expression patterns. Circles that overlap have a commonality, while circles that do not overlap do not share those traits (Fig. 5a).

The real-time quantitative PCR approached was used to verify the expression of 10 selected genes: TLR4, TLR7, TLR2-2, MyD88, TRAF6, IL1R2, CSF3R, IL8L1, TGFBR1, and IL18. The results confirmed a significant increase and the expression patterns of the selected genes, as was previously detected by the RNA-seq platform (Fig. 5b).

We used the principal component analysis (PCA) to visualize three-dimensional expression patterns of the RNA data sets (Fig. 5c).

\section{KEGG pathway analysis of differentially expressed genes}

The KEGG pathways have been significantly enriched in the subsets of up and down-regulated in each challenged group (FDR $<0.05$ ). The KEGG pathway enrichment analysis from the up-regulated genes was mainly involved in signaling pathways, and the KEGG pathway enrichment analysis from the down-regulated genes was clustered into cell growth and metabolic pathways (Table $2 \mathrm{~A}, \mathrm{~B}$, and $\mathrm{C}$ and 3,4 , and 5 ).

The gene expression heat map was used for the distinct analysis of gene expression pattern differences to relevant functions between all groups. In this method, the data is displayed in a grid, and each row represents a gene, and each column represents a sample. Gene expression was compared between lines. Based on the similarity of gene expression patterns, color changes (Fig. 5d).

Table 3. KEGG pathway analysis for up-regulated differentially expressed genes

\begin{tabular}{llll}
\hline ID & $\begin{array}{l}\text { KEGG } \\
\text { pathway-description }\end{array}$ & $\begin{array}{l}\text { Observed } \\
\text { gene } \\
\text { count }\end{array}$ & FDR \\
\hline gga00562 & $\begin{array}{l}\text { Inositol phosphate } \\
\text { metabolism }\end{array}$ & 24 & 0.00032 \\
gga04070 & $\begin{array}{l}\text { Phosphatidylinositol } \\
\text { signaling system }\end{array}$ & 27 & 0.00032 \\
gga04140 & Autophagy - animal & 31 & 0.00032 \\
gga04510 & Focal adhesion & 42 & 0.00032 \\
gga04371 & Apelin signaling pathway & 26 & 0.0039 \\
gga04144 & Endocytosis & 41 & 0.0088 \\
gga04150 & mTOR signaling pathway & 29 & 0.0088 \\
gga04810 & $\begin{array}{l}\text { Regulation of actin } \\
\text { cytoskeleton }\end{array}$ & 34 & 0.0098 \\
gga04910 & Insulin signaling pathway & 25 & 0.0141 \\
gga04512 & ECM-receptor interaction & 17 & 0.0204 \\
gga00564 & $\begin{array}{l}\text { Glycerophospholipid } \\
\text { metabolism }\end{array}$ & 19 & 0.0383 \\
gga04136 & Autophagy- other & 9 & 0.0383 \\
gga04520 & Adherens junction & 16 & 0.041 \\
\hline
\end{tabular}

Pathway analysis enrichment using DEGs between control and the group of infected with APEC. FDR $<0.05$ was used as a threshold to select significant KEGG pathways. 
Table 4. KEGG pathway analysis for up-regulated differentially expressed genes

\begin{tabular}{llll}
\hline ID & $\begin{array}{l}\text { KEGG } \\
\text { pathway-description }\end{array}$ & $\begin{array}{l}\text { Observed } \\
\text { gene } \\
\text { count }\end{array}$ & FDR \\
\hline gga00562 & $\begin{array}{l}\text { Inositol phosphate } \\
\text { metabolism }\end{array}$ & 26 & 0.0000438 \\
gga04510 & Focal adhesion & 44 & 0.0000438 \\
gga04140 & Autophagy - animal & 33 & 0.0000587 \\
gga04070 & $\begin{array}{l}\text { Phosphatidylinositol } \\
\text { signaling system }\end{array}$ & 28 & 0.00013 \\
gga04144 & Endocytosis & 45 & 0.0011 \\
gga04910 & Insulin signaling pathway & 28 & 0.0025 \\
gga04512 & ECM-receptor interaction & 19 & 0.0055 \\
gga04810 & $\begin{array}{l}\text { Regulation of actin } \\
\text { cytoskeleton }\end{array}$ & 34 & 0.0117 \\
gga04150 & mTOR signaling pathway & 28 & 0.0138 \\
gga04012 & ErbB signaling pathway & 18 & 0.0336 \\
gga04136 & Autophagy- other & 9 & 0.0407 \\
gga04371 & Apelin signaling pathway & 22 & 0.0407 \\
gga04010 & MAPK signaling pathway & 40 & 0.0416 \\
gga04120 & Ubiquitin mediated & 23 & 0.0416 \\
& proteolysis & & 0.0416 \\
gga04218 & Cellular senescence & 25 & 0.0416 \\
gga04520 & Adherens junction & 16 & \\
\hline
\end{tabular}

Pathway analysis enrichment using DEGs between control and the group of infected with IBV. FDR $<0.05$ was used as a threshold to select significant KEGG pathways.

\section{STRING analysis of the relationships between DEGs}

The STRING database of known and predicted protein interactions was used to predict interactions of the upregulated DEGs in the co-infected group. There were three main clusters in the STRING diagram. The apoptotic cluster contained 114 nodes associated with 579 edges (edges represent protein-protein interactions), the cytokinemediated signaling cluster contained 42 nodes associated with 123 edges, and the PAMPs (pathogen-associated molecular patterns) recognizing cluster contained 45 nodes associated with 201 edges (Fig. 6).

\section{Discussion}

The trachea plays a crucial role in the chickens' immune system, especially after challenging with respiratory pathogens. The purpose of our study was to determine the differences in chicken trachea transcriptome between non-infected chickens and chickens with individual IBV (Is-1494 like; GI-23) and APEC (serotype 078: K80)
Table 5. KEGG pathway analysis for up-regulated differentially expressed genes

\begin{tabular}{|c|c|c|c|}
\hline ID & $\begin{array}{l}\text { KEGG } \\
\text { pathway-description }\end{array}$ & $\begin{array}{l}\text { Observed } \\
\text { gene } \\
\text { count }\end{array}$ & FDR \\
\hline gga00562 & $\begin{array}{l}\text { Inositol phosphate } \\
\text { metabolism }\end{array}$ & 28 & 0.0000141 \\
\hline gga04070 & $\begin{array}{l}\text { Phosphatidylinositol } \\
\text { signaling system }\end{array}$ & 33 & 0.0000141 \\
\hline gga04140 & Autophagy - animal & 35 & 0.0000407 \\
\hline gga04510 & Focal adhesion & 45 & 0.0000551 \\
\hline gga04060 & $\begin{array}{l}\text { Cytokine-cytokine } \\
\text { receptor interaction }\end{array}$ & 42 & 0.00019 \\
\hline gga04144 & Endocytosis & 48 & 0.00066 \\
\hline gga04910 & Insulin signaling pathway & 31 & 0.00066 \\
\hline gga04217 & Necroptosis & 29 & 0.0011 \\
\hline gga04620 & $\begin{array}{l}\text { Toll-like receptor signaling } \\
\text { pathway }\end{array}$ & 23 & 0.0011 \\
\hline gga04621 & $\begin{array}{l}\text { NOD-like receptor } \\
\text { signaling pathway }\end{array}$ & 30 & 0.0011 \\
\hline gga04010 & MAPK signaling pathway & 49 & 0.0012 \\
\hline gga04210 & Apoptosis & 28 & 0.0012 \\
\hline gga04512 & ECM-receptor interaction & 21 & 0.0012 \\
\hline gga04810 & $\begin{array}{l}\text { Regulation of actin } \\
\text { cytoskeleton }\end{array}$ & 39 & 0.0012 \\
\hline gga04120 & $\begin{array}{l}\text { Ubiquitin mediated } \\
\text { proteolysis }\end{array}$ & 29 & 0.0014 \\
\hline gga05164 & Influenza A & 30 & 0.0014 \\
\hline gga04218 & Cellular senescence & 31 & 0.0017 \\
\hline gga04933 & $\begin{array}{l}\text { AGE-RAGE signaling } \\
\text { pathway in diabetic } \\
\text { complications }\end{array}$ & 23 & 0.0017 \\
\hline gga05132 & Salmonella infection & 19 & 0.0032 \\
\hline gga04012 & ErbB signaling pathway & 20 & 0.0072 \\
\hline gga04150 & mTOR signaling pathway & 28 & 0.0154 \\
\hline gga04514 & $\begin{array}{l}\text { Cell adhesion molecules } \\
\text { (CAMs) }\end{array}$ & 23 & 0.0154 \\
\hline
\end{tabular}

Pathway analysis enrichment using DEGs between control and coinfected group. FDR $<0.05$ was used as a threshold to select significant KEGG pathways.

infection and co-infection, with a focus on the use of the strand-specific NGS method to generate cDNA libraries for a robustly conserved transcript orientation and a more accurate determination of gene expression. In our investigation, the numbers of up-regulated genes were higher than of the down-regulated genes, and in the coinfected group, the induction of more immune pathways was shown.

The down-regulated pathways were ribosome and spliceosome in three groups, oxidative phosphorylation in the 


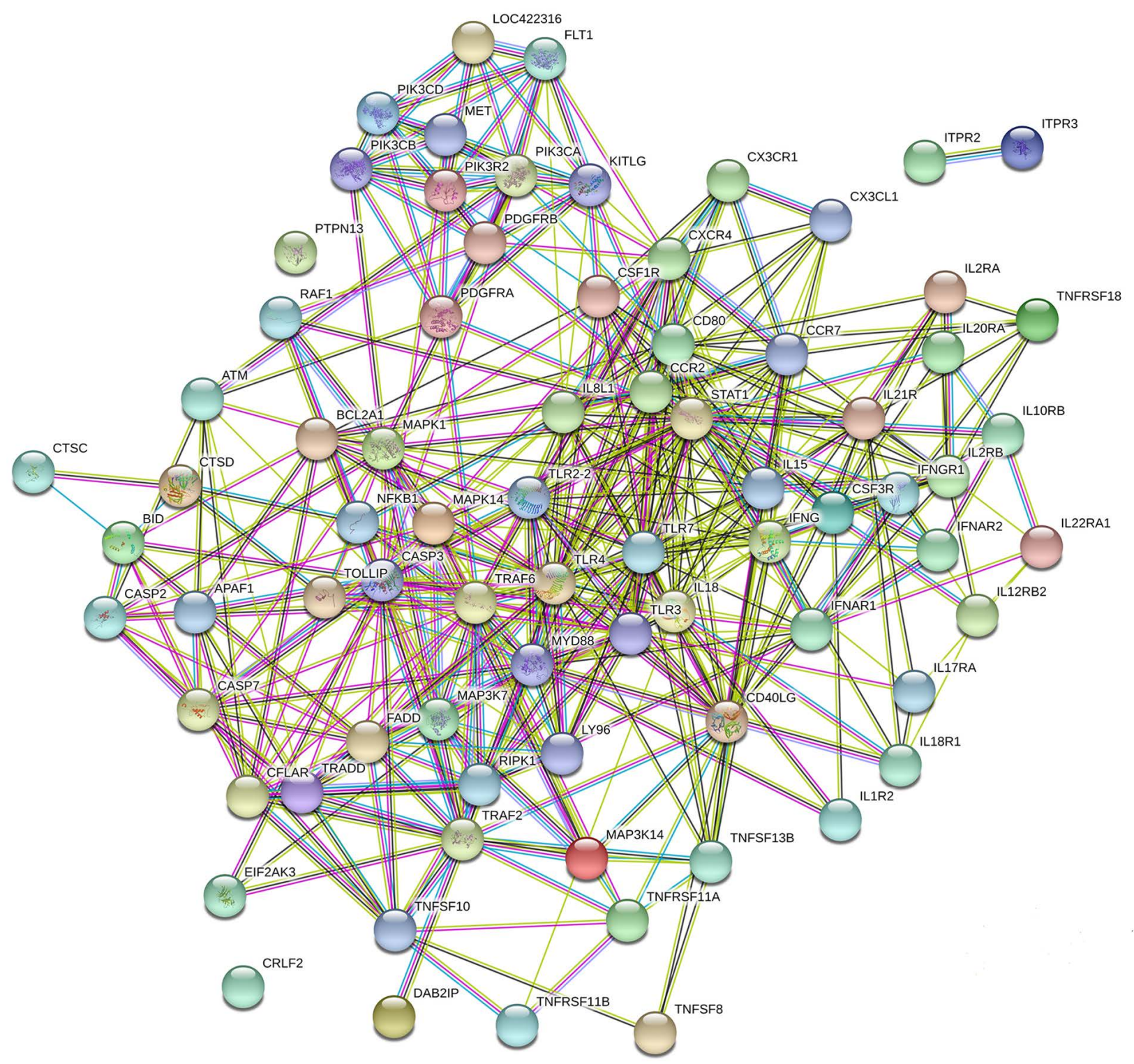

Fig. 6

STRING analysis of the relationship between differentially expressed genes (FDR $<0.05$ ) related to the apoptotic pathways, the cytokine-mediated signaling pathways, and the PAMPs recognized pathways in up-regulated genes in the co-infected group The network nodes represent the proteins encoded by the DEGs, and the edges represent protein-protein interactions.

group infected with IBV and in the co-infected group, and Glutathione metabolism and Metabolic pathways only in the co-infected group; the genes were clustered into protein production, energy production, and metabolic processes.

The pathways of signaling processes, including changes in cell shape, migration, proliferation, cell metabolism, growth, proliferation, and survival, consist of inositol phosphate metabolism, focal adhesion, insulin signaling pathway, ECM-receptor interaction, regulation of actin cytoskeleton, mTOR signaling pathway, adherence junction were up-regulated in all three groups.

Apelin signaling pathway was up-regulated only in the group infected with IBV and infected with APEC. Erbb signaling pathway and MAPK signaling pathway were upregulated only in the co-infected group and in the group infected with IBV. MAPK signaling pathway is one of the more important pathways and is an essential pathway to promote inflammatory response through macrophage activation (Khatri and Sharma, 2006). Calcium signaling 
pathways and cell adhesion molecules (CAMs) were upregulated only in the co-infected group (Tables 4 and 5). This suggests that host cell receptors might recognize the organisms and secrete cytokines to cope with pathogens invasion.

Lipopolysaccharide(LPS), bacteria, and virus infection stimulate signal transduction pathways that play roles in the regulation of many events such as cell proliferation and differentiation, cytokine production, apoptosis, and cell death, cell survival, and stimulation of immune responses (Zhang and Liu, 2002; Hynes et al., 2013). PRRs rapidly recognize infectious agents and affect biological events by identifying PAMPs (Motta et al., 2015). They initiate both innate and adaptive immune responses. The PAMPs that enter the cell via phagocytosis or pores and DAMPs (damage-associated molecular patterns) are identified by NLRs (Chhabra et al., 2015). In the present study, the following genes were up-regulated in the co-infected group: ANTXR2, CYBB, FADD, IFNAR1, IFNAR2, IL18, IL8L1, ITPR2, ITPR3, JAK1, MAP3K7, MAPK1, MAPK14, MCU, MFN2, MYD88, NAMPT, NFKB1, NOD1, P2RX7, PLCB2, PSTPIP1, RIPK1, STAT1, TLR4, TNFAIP3, TRAF2, TRAF5, TRAF6, and TRPV2. TLRs signaling pathway and RLRs signaling pathway were up-regulated in co-infected group too. These genes were associated with RLRs according to our results: AZI2, CYLD, DDX3X, FADD, IFIH1, IL8L1, MAP3K7, MAPK14, NFKB1, RIPK1, TRADD, TRAF2, and TRAF6.

The TLR2 recognizes peptidoglycan; TLR4 binds LPS, TLR5 recognizes flagellin, and TLR21 recognizes unmethylated CpG DNA that is found in bacteria (Keestra et al., 2010). In chickens, TLR 3 and 7 recognize RNA viral products (Cook et al., 2012; Chen et al., 2013). In this study, expression of several TLRs was documented through the up-regulation of CD80, FADD, IFNAR1, IFNAR2, IL8L1, LY96, MAP3K7, MAPK1, MAPK14, MYD88, NFKB1, PIK3CA, PIK3CB, PIK3CD, PIK3R2, RIPK1, STAT1, TLR2-2, TLR3, TLR4, TLR7, TOLLIP, TRAF6 in the co-infected group. In a preview study, TLR1LA, TLR1LB, TLR2, TLR3, and TLR7 gene expression was significantly up-regulated in the tracheal epithelial cells of 3-weeks-old chickens immunized with attenuated IBV Massachusetts (IBV-Mass) strain (Guo et al., 2008). Also, the up-regulation of TLR3 and TLR7 mRNA was identified in the trachea and lung during viral infections (Miettinen et al., 2001; Wang et al., 2006). In the lungs, APEC strains are likely to be recognized through TLRs expressed by epithelial and resident phagocytic cells, which would drive inflammatory mediators' production and stimulate phagocytosis (Guabiraba and Schouler, 2015).

TLR3 signaling pathway produces antiviral type I interferon IFN- $\alpha$ and $-\beta$, and the TLR7 signaling pathway produces pro-inflammatory cytokines (Guillot et al., 2005). IL1B has an important role in chemotaxis, stimulation of the cellular response, and infiltration of immune cells, such as macrophages, to the site of infection (Babcock et al., 2008). In chickens, IL8 is known for its chemotactic activity for macrophages and heterophils, and, after in vivo interaction of respiratory epithelial cells with APEC infections, an increase in macrophages was detected (Mol et al., 2019). Macrophages are a source of nitric oxide (NO) in defense against microbial infections (Ariaans et al., 2008) and play a similar role in bacterial infection of the chicken. They also play an important role as antigenpresenting cells (Wu and Kaiser, 2011) and in producing important effectors and regulators of immunity (He et al., 2011). As observed in our histopathology findings, the infiltration of inflammatory cells was in the co-infected group; however, in 2 groups with individual infections, only moderate pathology was induced.

It has been found that cytokine-cytokine receptor interaction was up-regulated in the co-infected group. Upon infection, PRRs initiate signaling cascades that, as a result, activate the anti-infection innate immune response (Diebold, 2010). Analysis of DEGs revealed expression of BMPR1A, BMPR2, CCR2, CCR7, CD40LG, CRLF2, CSF1R, CSF3R, CX3CL1, CX3CR1, CXCR4, FLT1, IFNAR1, IFNAR2, IFNG, IFNGR1, IL10RB, IL12RB2, IL15, IL17RA, IL18, IL18R1, IL1R2, IL20RA, IL21R, IL22RA1, IL2RA, IL2RB, IL8L1, KITLG, LOC422316, MET, PDGFRA, PDGFRB, TGFBR1, TGFBR2, TNFRSF11A, TNFRSF11B, TNFRSF18, TNFSF10, TNFSF13B, and TNFSF8 in our study. The production of these proinflammatory cytokines and chemokines supports macrophages' involvement, and activated macrophages produce a variety of pro-inflammatory cytokines and modulate the inflammatory reaction via the up-regulation of a few inflammatory cytokines (Holmes and Darbyshire, 1978). In viral or bacterial infection, the organs can be damaged by excessive activation of the inflammatory response using the cytokine storm. Thus, it seems that the recruitment of inflammatory cells and macrophages' activation is reasonable to the development of damage in chickens suffering from an infection. The up-regulation of mRNA levels of pro-inflammatory cytokines IL6, IL1B, IL10R, IL2, IL4, IL7, IL9, IL15, IL21, IL17RA, CCL4, CCL20, CCL17, and CCL19 in the trachea following infection with IBV has been previously shown (Cong et al., 2013; Holmes and Darbyshire, 1978). The pro-inflammatory cytokines IL4, IL10, and IFN- $\gamma$ receptor 2 have previously been found in APEC-infected birds (Sandford et al., 2012). Changes in interleukin receptor genes have been noted in response to pathogen challenge (Crowley et al., 2011).

It has been shown that autophagy was up-regulated in all three infected groups. Autophagy is a key physiopathological process with a complex relationship with cell death and survival (Hu et al., 2015). Moreover, in the co-infected group, apoptosis and necroptosis were also 
up-regulated. One of the most important host antiviral responses is the induction of apoptosis. Induction of cell death following infection of target cells can effectively limit virus replication (Hay and Kannourakis, 2002). As a result of the entry of both pathogenic and non-pathogenic strains of avian E. coli in the avian lung, the production of focal infections was shown, which can induce inflammatory mediators and cell death in the infected areas (Horn et al., 2012). In this study, genes implicated in apoptosis signaling pathway were mostly up-regulated and involved as survival factors; including APAF1, ATM, BCL2A1, BID, CASP2, CASP3, CASP7, CFLAR, CTSC, CTSD, DAB2IP, EIF2AK3, FADD, ITPR2, ITPR3, MAP3K14, MAPK1, NFKB1, PIK3CA, PIK3CB, PIK3CD, PIK3R2, PTPN13, RAF1, RIPK1, TNFSF10, TRADD, and TRAF2. Studies showed that IBV induced cell cycle arrest and apoptosis in infected cells (Li et al., 2007; Chhabra et al., 2015). A strain of APEC (APEC17) was previously shown to activate caspase $3 / 7$ in macrophages, thereby inducing apoptosis (Guabiraba and Schouler, 2015).

AGE-RAGE signaling pathway, involved in diabetic complications, and FoxO signaling pathway were also up-regulated in the co-infected group. AGE/RAGE signaling leads to the activation of multiple intracellular signal pathways involving NADPH oxidase, protein kinase $\mathrm{C}$, and MAPKs, resulting in NFkB activity. It has been shown that as a result of activation of NFkB, the IL1, IL6, and TNFalpha, a variety of atherosclerosis-related genes, including VCAM1, tissue factor, VEGF, and RAGE are expressed (Kay et al., 2016). Ubiquitin-mediated proteolysis was only upregulated in the co-infected group and infected with only IBV. These data suggest that activation of apoptosis and suppression of innate immune response occurred in the trachea in co-infection with IBV and APEC in the early phase of infection. VEGF has several isoforms that bind to different receptors such as FLT1, KDR, and NRP1, which can promote angiogenesis through activation of a kinase cascade, including RAS and MAPK (Maitland et al., 2010). Studies have shown that in viral infections, both systemic (IgM and IgG) and mucosal (IgA) antibodies effectively clear the circulating virus in the host (Darbyshire and Peters, 1985). Also, IgA is the major immunoglobulin molecule for the mucosal response that plays an important role in protecting against infection at tracheal or other mucosal points of viral entry (Caron, 2010).

The novel experiment presented in this study characterized changes in the chicken trachea transcriptome associated with two respiratory pathogens, IBV (IS-1494 like; GI-23) and APEC (serotype O78: K80) infection and coinfection, in the early phase of infection. In the previous studies with chickens infected with the virus followed by a bacterial infection, it was shown that it reflects an especially severe form of the naturally occurring disease
(Cook et al., 1986). According to our analysis of transcriptome and the histopathological findings, regulation of gene expression among the three treatment groups at the early stage of infection was different in the co-infected group, and it induced an innate immune response within the trachea through increased mRNA expression of PPRs, pro-inflammatory cytokines, and anti-viral IFNs, and through an increase in the number of infiltrating macrophages to the trachea, with some of the innate genes being up-regulated following infection. In contrast, in the two groups infected with individual pathogens, only a mild immune response was observed at the early stage of infection.

Acknowledgments. The authors gratefully thank Iran National Science Foundation for supporting this study by granting its project (No. 95836652).

\section{References}

Ariaans MP, Matthijs MG, van Haarlem D, van de Haar P, van Eck JH, Hensen EJ, Vervelde L (2008): The role of phagocytic cells in enhanced susceptibility of broilers to colibacillosis after infectious bronchitis virus infection. Vet. Immunol. Immunopathol. 123, 240-250. https://doi.org/10.1016/j.vetimm.2008.02.003

Asif M, Lowenthal JW, Ford ME, Schat KA, Kimpton WG, Bean AG (2007) Interleukin-6 expression after infectious bronchitis virus infection in chickens. Viral Immunol. 20, 479-486. https://doi.org/10.1089/vim.2006.0109

Babcock AA, Toft-Hansen H, Owens T (2008): Signaling through MyD88 regulates leukocyte recruitment after brain injury. J. Immunol. 181, 6481-6490. https://doi. org/10.4049/jimmunol.181.9.6481

Caron L (2010): Etiology and immunology of infectious bronchitis virus. Braz. J. Poult. Sci. 12, 115-119. https://doi. org/10.1590/S1516-635X2010000200007

Chen S, Cheng A, Wang M (2013): Innate sensing of viruses by pattern recognition receptors in birds. Vet. Res. 44, 82 . https://doi.org/10.1186/1297-9716-44-82

Chhabra R, Chantrey J, Ganapathy K (2015): Immune responses to virulent and vaccine strains of infectious bronchitis viruses in chickens. Viral immunol. 28, 478-488. https://doi.org/10.1089/vim.2015.0027

Cong F, Liu X, Han Z, Shao Y, Kong X, Liu S (2013): Transcriptome analysis of chicken kidney tissues following coronavirus avian infectious bronchitis virus infection. BMC Genomics 14, 743. https://doi.org/10.1186/14712164-14-743

Cook JK, Jackwood M, Jones R (2012): The long view: 40 years of infectious bronchitis research. Avian Pathol. 41, 239-250.https://doi.org/10.1080/03079457.2012.680432

Cook JK, Smith HW, Huggins M (1986): Infectious bronchitis immunity: its study in chickens experimentally infected with mixtures of infectious bronchitis virus and 
Escherichia coli. J. Gen. Virol.67,1427-1434. https://doi. org/10.1099/0022-1317-67-7-1427

Crowley TM, Haring VR, Moore R (2011): Chicken anemia virus: an understanding of the in-vitro host response over time. Viral Immunol. 24, 3-9. https://doi.org/10.1089/ vim.2010.0064

Darbyshire J, Peters R (1985): Humoral antibody response and assessment of protection following primary vaccination of chicks with maternally derived antibody against avian infectious bronchitis virus. Res. Vet. Sci. 38,14-21. https://doi.org/10.1016/S0034-5288(18)31841-1

Diebold S (2010): Innate recognition of viruses. Immunol. Lett. 128, 17-20. https://doi.org/10.1016/j.imlet.2009.09.010

Guabiraba R, Schouler C (2015): Avian colibacillosis: still many black holes. FEMS Microbiol. Lett. 362, fnv118. https:// doi.org/10.1093/femsle/fnv118

Guillot L, Le Goffic R, Bloch S, Escriou N, Akira S, Chignard M, Si-Tahar M (2005): Involvement of toll-like receptor 3 in the immune response of lung epithelial cells to doublestranded RNA and influenza A virus. J. Biol. Chem. 280, 5571-5580. https://doi.org/10.1074/jbc.M410592200

Guo X, Rosa AJ, Chen D-G, Wang X (2008): Molecular mechanisms of primary and secondary mucosal immunity using avian infectious bronchitis virus as a model system. Vet. Immunol. Immunopathol. 121, 332-343. https://doi.org/10.1016/j.vetimm.2007.09.016

Hay S, Kannourakis G (2002): A time to kill: viral manipulation of the cell death program. J. Gen. Virol. 83, 1547-1564. https://doi.org/10.1099/0022-1317-83-7-1547

He H, Genovese KJ, Kogut MH (2011): Modulation of chicken macrophage effector function by TH1/TH2 cytokines. Cytokine 53, 363-369. https://doi.org/10.1016/j. cyto.2010.12.009

Holmes H, Darbyshire J (1978): Induction of chicken interferon by avian infectious bronchitis virus. Res. Vet. Sci. 25, 178-181. https://doi.org/10.1016/S0034-5288(18)32975-8

Horn F, Corrêa AMR, Barbieri NL, Glodde S, Weyrauch KD, Kaspers B, Driemeier D, Ewers C, Wieler LH (2012): Infections with avian pathogenic and fecal Escherichia coli strains display similar lung histopathology and macrophage apoptosis. PloS One 7, e41031. https://doi. org/10.1371/journal.pone.0041031

Hu B, Zhang Y, Jia L, Wu H, Fan C, Sun Y, Ye C, Liao M, Zhou J (2015): Binding of the pathogen receptor HSP90AA1 to avibirnavirus VP2 induces autophagy by inactivating the AKT-MTOR pathway. Autophagy 11, 503-515. https://doi.org/10.1080/15548627.2015.1017184

Hynes NE, Ingham PW, Lim WA, Marshall CJ, Massagué J, Pawson T (2013): Signalling change: signal transduction through the decades. Nat. Rev. Mol. Cell Biol. 14, 393-398. https://doi.org/10.1038/nrm3581

Jackwood MW (2012): Review of infectious bronchitis virus around the world. Avian Dis. 56, 634-641. https://doi. org/10.1637/10227-043012-Review.1

Kameka AM, Haddadi S, Kim DS, Cork SC, Abdul-Careem MF (2014): Induction of innate immune response following infectious bronchitis corona virus infection in the respiratory tract of chickens. Virology 450,114-121. https://doi.org/10.1016/j.virol.2013.12.001

Kay AM, Simpson CL, Stewart JA (2016): The role of AGE/ RAGE signaling in diabetes-mediated vascular calcification. J. Diabetes Res. 2016, 6809703. https://doi. org $/ 10.1155 / 2016 / 6809703$

Keestra AM, de Zoete MR, Bouwman LI, van Putten JP (2010): Chicken TLR21 is an innate CpG DNA receptor distinct from mammalian TLR9. J. Immunol. 185, 460-467. https://doi.org/10.4049/jimmunol.0901921

Khatri M, Sharma JM (2006): Infectious bursal disease virus infection induces macrophage activation via p38 MAPK and NF-кB pathways. Virus Res. 118, 70-77. https://doi. org/10.1016/i.virusres.2005.11.015

Kruth SA (1998): Biological response modifiers: interferons, interleukins, recombinant products, liposomal products. Vet. Clin. North Am. Small Anim. Pract. 28, 269295. https://doi.org/10.1016/S0195-5616(98)82005-6

Li FQ, Tam JP, Liu DX (2007): Cell cycle arrest and apoptosis induced by the coronavirus infectious bronchitis virus in the absence of p53. Virology 365, 435-445. https:// doi.org/10.1016/j.virol.2007.04.015

Lisowska A, Sajewicz-Krukowska J, Fusaro A, Pikula A, Domanska-Blicharz K (2017): First characterization of a MiddleEast GI-23 lineage (Var2-like) of infectious bronchitis virus in Europe. Virus Res. 242, 43-48. https://doi. org/10.1016/j.virusres.2017.09.010

Maitland ML, Lou XJ, Ramirez J, Desai AA, Berlin DS, McLeod HL, Weichselbaum RR, Ratain MJ, Altman RB, Klein TE (2010): Vascular endothelial growth factor pathway. Pharmacogenet. Genomics 20, 346-349. https://doi. org/10.1097/FPC.0b013e3283364ed7

Miettinen M, Sareneva T, Julkunen I, Matikainen S (2001): IFNs activate toll-like receptor gene expression in viral infections. Genes Immun. 2, 349-355. https://doi. org/10.1038/sj.gene.6363791

Mol N, Peng L, Esnault E, Quéré P, Haagsman HP, Veldhuizen EJ (2019): Avian pathogenic Escherichia coli infection of a chicken lung epithelial cell line. Vet. Immunol. Immunopathol. 210, 55-59. https://doi.org/10.1016/i. vetimm.2019.03.007

Motta V, Soares F, Sun T, Philpott DJ (2015): NOD-like receptors: versatile cytosolic sentinels. Physiol. Rev. 95, 149-178. https://doi.org/10.1152/physrev.00009.2014

Najafi H, Ghalyanchi AL, Hashemzadeh M, Madadgar O, Karimi V, Farahani R, Abdollahi H, Maghsoudsloo H, Seifouri P (2016): Pathogenicity characteristics of an Iranian variant-2 (IS-1494) like infectious bronchitis virus in experimentally infected SPF chickens. Acta Virol. 60, 393-399. https://doi.org/10.4149/av $201604 \quad 393$

Paul MS, Brisbin JT, Abdul-Careem MF, Sharif S (2013): Immunostimulatory properties of Toll-like receptor ligands in chickens. Vet. Immunol. Immunopathol.152,191-199. https://doi.org/10.1016/j.vetimm.2012.10.013

Peighambari S, Julian R, Gyles C (2000): Experimental Escherichia coli respiratory infection in broilers. Avian Dis. 44, 759-769. https://doi.org/10.2307/1593047 
Sandford EE, Orr M, Shelby M, Li X, Zhou H, Johnson TJ, Kariyawasam S, Liu P, Nolan LK, Lamont SJ (2012): Leukocyte transcriptome from chickens infected with avian pathogenic Escherichia coli identifies pathways associated with resistance. Results Immunol. 2, 44-53. https://doi.org/10.1016/j.rinim.2012.02.003

Wang X, Rosa AJ, Oliverira HN, Rosa GJ, Guo X, Travnicek M, Girshick T (2006): Transcriptome of local innate and adaptive immunity during early phase of infectious bronchitis viral infection. Viral Immunol. 19, 768-774. https://doi.org/10.1089/vim.2006.19.768
Westermann AJ, Gorski SA, Vogel J (2012): Dual RNA-seq of pathogen and host. Nat. Rev. Microbiol. 10, 618-630. https://doi.org/10.1038/nrmicro2852

Wu Z, Kaiser P (2011): Antigen presenting cells in a non-mammalian model system, the chicken. Immunobiology 216, 1177-1183. https://doi.org/10.1016/j.imbio.2011.05.012

Zhang W, Liu HT (2002): MAPK signal pathways in the regulation of cell proliferation in mammalian cells. Cell Res. 12, 9-18. https://doi.org/10.1038/sj.cr.7290105 